\title{
In Situ X-ray Absorption Near-Edge Structure Spectroscopy Reveals Arsenic Ligation in Rice
}

\author{
Benjamin C. Bostick ${ }^{1, *}$, YATING SheN ${ }^{1,2}$, Elizabeth \\ WIITA $^{1,3}$, ATHENA A. NGHIEM ${ }^{1}$, JiNGYU LIU ${ }^{4}$, \\ KONGKEA PHAN ${ }^{5}$ AND YAN ZHENG ${ }^{1,4}$, \\ ${ }^{1}$ Lamont-Doherty Earth Observatory, Columbia University, \\ Palisades, New York, 10964, USA, (*correspondence: \\ bostick@ldeo.columbia.edu) \\ ${ }^{2}$ National Research Center for Geoanalysis (NRCGA), \\ Beijing, 100037, China \\ ${ }^{3}$ Barnard College, Dept. Chemistry, New York, NY, USA \\ ${ }^{4}$ South Univ. Sci. Technol. of China, Shenzhen, China \\ ${ }^{5}$ International Univ, Phnom Penh, Cambodia
}

Arsenic is often abundant in rice because of the physiology of rice plants, and the flooded environments typical of rice production. The exposure to this source of arsenic in food is believed to represent the largest single source of toxic arsenic for most humans. The risks posed by As depend strongly on the chemical speciation of arsenic. Inorganic inorganic arsenic(III) and (V) is regarded as much more toxic than the methylated metabolites, mono- and dimethylarsenic (DMA). Arsenosugars and arsenobetaine are abundant in seafood but are much less toxic still. Most studies of arsenic speciation in rice (and water) use chromatographic separations that preserve the extent of methylation, but these methods do not preserve the coordination state, nor the oxidation state of As. Here we report an in situ measurements of As speciation and distribution in rice using the combination of X-ray absorption spectroscopy and microprobe X-ray fluorescence spectroscopy. Using a combination of white line position and intensity, we can identify specific ligands that coordinate As, and their coordination number. We successfully measure As speciation in rice samples with total As levels as low as $50 \mu \mathrm{gAs} / \mathrm{kg}$, distributed fairly evenly throughout the grain. These results indicate that much of the As found in rice is present as thiolbound $\mathrm{As}(\mathrm{III})$ and $\mathrm{DMA}(\mathrm{V})$ rather than the oxyacid or oxyanion forms identified following extraction. These complexes may have widely different bioavailability or toxicity than is assumed based on aqueous speciation. Similar As-thiol complexes have recently been identified in rice [1] and rice paddies [2]. Given the prevalence of thiol complexation, we suggest that future studies consider these forms of As in the environment and rice plants, and their transfer through the environment.

[1] Kerl et al. (2019), Env. Sci. Tech. 53, 5787-5796. [2] Wang et al. (2020) Nature Geosci. 15, 403-413. 